\title{
Hierarchical Task Analysis to Medication Administration Errors
}

Authors:

Rhonda Lane

Neville A Stanton,

David Harrison

Brunel University

Department of Design and Information Systems

Kingston Lane

Uxbridge

UB8 3PH

Tel: 01895274000

Email:Rhonda.Lane@brunel.ac.uk 


\section{Applying Hierarchical Task Analysis to Medication}

\section{Administration Errors}

Rhonda Lane, Neville A Stanton, David Harrison

Brunel University

\section{Abstract}

This paper demonstrates how the human error identification technique SHERPA can be applied to the task of administering drugs to hospital patients. Human error identification techniques have been used in high-risk environments for some time. Drugs administration is a complex and risky process with many opportunities for error. The purpose of using SHERPA is to identify where in the process errors occur and to suggest the most suitable design solutions to mitigate these errors.

\section{Medical Error}

Improving the quality and safety of patient care has become an increasingly high priority in both UK and across the world (Department of Health, 2001). Medical errors affect 850,000 people in the UK each year often leading to patients spending extended time in hospital or in community care. These errors cost the NHS up to $£ 2$ billion in additional treatment and the cost of clinical negligence settlements is about $£ 400$ million (DOH, 2000).

Medical error is also a significant cause of death in the United States. The Harvard Medical Practice Study (Brennan et al, 1991) using data from 30,121 randomly selected patient records identified a total of 1278 adverse events. From this figure it was estimated that adverse events occurred in 3.7\% of hospitalisations and of these adverse 
events $27.6 \%$ were due to negligence. (An adverse event is an actual or potential unintended injury resulting from any form of medical treatment rather than the disease process.) For $70 \%$ of patients the adverse event led to slight or temporary disability. Disability was permanent in $7 \%$ of patients and in $13.6 \%$ of cases the outcome was fatal.

\section{Medication error}

Many of the major medical error studies have highlighted medication errors as a cause of adverse events suffered by patients (Bates et al, 1995; Leape et al, 1995; Brennan 1991 Kohn et al, 1999). Approximately a quarter of all claims to the Medical Defence Union relate to drugs. Some of these claims may also be due to adverse drug events (ADEs). An ADE is an injury caused by medications (Leape et al, 1995). It may or may not be the result of error. The Audit Commission Report, A Spoonful of Sugar (2001), reports that about 1200 deaths occur in the UK each year due to medication related problems. Of these, 200 are the result of medication errors.

Ferner and Aronson (2000) define a medication error as: "a failure in a drug treatment process that leads to or has the potential to lead to harm to the patient.“ The definition most commonly used is that given by the National Co-ordinating Council for Medication Error Reporting and Prevention (NCC MERP) in the USA:

"...any preventable event that may cause or lead to inappropriate medication use or patient harm, while the medication is in the control of the healthcare professional, patient or consumer. Such events may be related to professional practice, healthcare products, procedures, and systems including: prescribing; order communication; product 
labelling, packaging and nomenclature; compounding; dispensing;

distribution; administration; education; monitoring and use."

American systems for monitoring medication errors are more systematic than those in the UK, though this is changing. The United States Pharmacopeia (USP) in association with the Institute for Safe Medication Practices (ISMP) has monitored medication errors since 1991. Since 1998 the USP has collected information about medication error through MedMarx, an internet-based reporting system. During 2000 a total of 41,296 error reports were received of which $42 \%$ were errors in administering drugs (Figure 1). The other errors occurred during: prescribing (13\%), transcribing (27\%), dispensing (17\%) and monitoring (1\%). Sixty three percent of the errors were not intercepted before they reached the patient and 3\% of errors caused harm or a fatality.

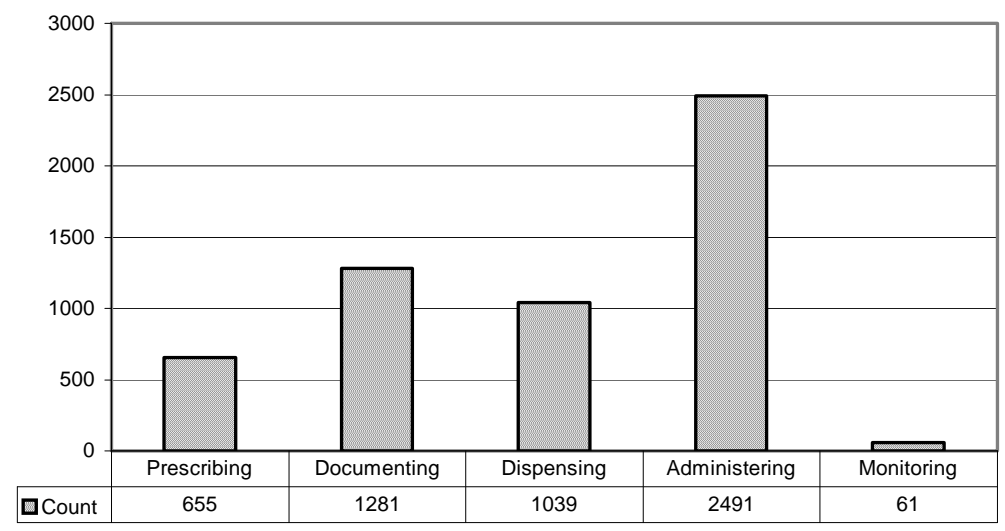

Figure 1: Node where most errors have been reported (USP, 2000)

A study by Philips et al (2001) examined 5366 medication error reports from across the world submitted between1993 and 1998. Patients suffered serious outcomes in 68.2\% of cases and 469 (9.8\%) people died. The most common types of errors resulting in patient death were: 
Improper dose

Wrong drug

Wrong administration route

Wrong strength
$40.9 \%$

$16 \%$

$9.5 \%$

$5.7 \%$

The most common causes of error were deficits in performance and knowledge (44\%) and errors in communication (15.8\%).

\section{Causes of medication error}

Medication use is complex and is dependent on the successful interaction of health professionals functioning within different disciplines. Errors can occur at any one of the five main stages of prescribing, documenting, dispensing or preparation, administering and monitoring. Wolf (1993) has pointed out that nurses make medication errors regardless of their speciality and that errors occurred on medical and surgical floors, postpartum units, emergency units and medical and surgical intensive care units.

The responsibility for the error is often placed on the nurse, as she or he is the last person in the drug administration chain. A parallel can be drawn with aviation. Pilots used to be held responsible for aviation accidents until analysis revealed other factors were highly significant. The reality is that there are many different causes of medication errors and they often combine to cause the incident. For instance, commonly reported reasons due to organisational factors are: workload, constant interruptions, shift patterns (Ferner, 1995); communication failures (Fiesta, 1998) and long working hours (DOH. 2000b). Thornton et al (1999) cite lack of proficiency in calculation of drug doses, dilutions and rates of administration; incorrect preparation or compounding of drugs; inappropriate drug selection and using incorrect routes of 
administration. Leape et al (1995) in addition to the above, identified lack of information about the patient; rule violations (failure to follow accepted and wellestablished procedures); slips and memory lapses in which the individual 'knew better' and could not explain why the error occurred and drug stock or delivery problems.

\section{The Drug administration Task}

In the UK many hospitals use a ward based pharmacy system and about $80 \%$ of medications administered are held as ward stock. These drugs are the most frequently used and are stocked in bottles of 50 or 100 tablets or capsules. Non-stock items are stored in a locked trolley. Physicians use the patient's chart to indicate to the nurse which medications the patient is to receive. This is kept with the patient and used to record drug administration. The order includes the drug name, the dose and the drug administration round when it is to be administered. Nurses make a drug administration round with a trolley four to six times daily depending on the ward. Each dose is recorded on the medication chart, which usually allows 14 days of documentation. This allows the patient's most recent drug history to be viewed. Pharmacists visit their designated wards daily to review all patient charts performing a clinical and supply function. If a drug is ordered that is not held as ward stock, the pharmacist makes a note on the medication chart and a supply sufficient for several days is dispensed with the patient's name on the container (Dean et al, 1995). The other system that is increasingly used is the patient's own drug system in which the patient continues to use medication they would normally use at home. This is kept in a locker at the bedside.

Giving medications to patients is a fundamental nursing role. It is also a complex activity that carries a high risk of error, as the involvement of different health care professionals means that errors may occur at any stage of the process (Hand and Barber, 
2000). Nurses seek to give medications correctly or perfectly. However their efforts are often confounded by poorly written prescriptions, constant interruptions, conflicting demands and high workloads.

\section{Addressing the problem}

Failure mode and effects analysis (FMEA) has been applied to drug administration as a process of continuous quality improvement and is usually carried out by an interdisciplinary group of healthcare professionals (Cohen, 1994). A FMEA anticipates what errors can be made and what the results will be. Thus for each medication the analyst will ask what will happen when someone mistakes a drug package for something else; uses the wrong amount of drug, gives the drug to the wrong patient, gives the drug by the wrong route, gives the wrong rate of a drug and so on. The analyst then goes on to consider how best to prevent incorrect action being completed or to minimise their ability to cause an adverse event if they are completed.

The National Patient Safety Agency (NPSA) has set up a reporting and learning system for general and medication errors occurring in the UK. It has also implemented the Root Cause Analysis system as a way of systematically identifying the factors affecting clinical error.

\section{Applying HTA to drug administration}

Many of the above studies have systematically analysed the context of drug administration. However, there is still merit in analysing the process at task level to assess how best to reduce errors through design solutions. Carrying out some form of human error analysis enables us to gain an understanding of how human interaction with drug administration tasks might lead to incidents. A starting point might be to 
explore the procedure for getting drugs to patients and then to examine the task steps, the equipment used and the relationships between these factors.

Task analysis is a way of breaking the task down into its component parts. It is also a useful way of looking at how people interact with equipment and with various aspects of their working environment. Task analyses can be organised in a variety of ways and a commonly used format is to arrange information in a hierarchical form.

A hierarchical task analysis (HTA) for the drug administration process is shown in the Appendix A. The HTA was drawn up by the lead author who trained as a hospital pharmacy technician. It was reviewed by two nurses, a hospital risk manager and two patient safety experts.

The top-level goal of the system is to deliver drugs to the patient. The task steps necessary to do this are listed as tasks 1 to 3 on the next level of the hierarchy. Plan 0 indicates the activities or sub-goals that should be carried out in order to achieve the goal. These activities are further broken down into operations at the lower levels. The order in which these are carried out is determined by the plan.

\section{SHERPA}

The Systematic Human Error Reduction and Prediction Approach (SHERPA) (Embrey 1986) was originally developed for use in the nuclear industry. It utilises a computerized question and answer structure to identify likely errors in the task analysis. The errors identified are based on the skill, rule, knowledge (SRK) model devised by Rasmussen et al (1981) and Reason’s (1987) generic error modelling system (GEMS). The approach attempts to link error reduction measures to underlying causes of human 
error. This human identification error technique allows the analyst to define the information that is useful for error reduction strategies. It should be pointed out that experienced nurses are often able to recover their errors by correcting them before the patient suffers harm (Wolf et al, 1997; Baker, 1997).

\section{Error Predictions}

SHERPA uses the bottom level actions of the HTA as its inputs. These are the operations or task steps carried out to achieve the higher-level goal. The operations are evaluated for potential error using the human error taxonomy shown in Table 1. The types of error that may occur fall into one of five behaviour categories: action, checking, retrieval, communication and selection. Each error type in the taxonomy is coded and is associated with an error mode.

The task steps from the HTA are examined in turn and classified into one of the error types. The most likely error modes associated with that operation are considered. For example, the task step 1.1.1 in the HTA “check patient bed” (Appendix A) is classified as a checking activity. Looking at the associated checking error modes in Table 1, only the most credible errors for the task step are taken into account. It is possible that whilst searching for a patient chart a nurse may fail to check the area around a patient's bed or may be called away to attend to another patient and so not complete the search. In nursing terms these are not strictly errors but within the SHERPA system these actions would prevent the goal of finding patient chart being achieved.

The results of the SHERPA analysis are recorded in tabular form (Appendix B). In the first column the number of the task step is listed (1.1.1). The error mode C1 is entered 
in the second column. This denotes a check has been missed (Table 1). In the third column headed "description” an outline of the error is entered. In this case the

Table 1: SHERPA Error Modes

\begin{tabular}{lll}
\hline Error type & Code & Error Mode \\
\hline Action Errors & A1 & Operation too long/short \\
A2 & Operation mistimed \\
A3 & Operation in wrong direction \\
A4 & Operation too little/much \\
A5 & Misalign \\
& A6 & Right operation on wrong object \\
A7 & Wrong operation on right object \\
A8 & Operation omitted \\
& A9 & Operation incomplete \\
& A10 & Wrong operation on wrong object \\
& C1 & Check omitted \\
& C2 & Check incomplete \\
C3 & Right check on wrong object \\
Checking Errors & C4 & Wrong check on right object \\
& C5 & Check mistimed \\
& C6 & Wrong check on wrong object \\
& & \\
R1 & Information not obtained \\
R2 & Wrong information obtained \\
R3 & Information retrieval incomplete \\
& I1 & Information not communicated \\
& I2 & Wrong information communicated \\
I3 & Information communication incomplete \\
& S1 & Selection omitted \\
S2 & Wrong selection made \\
\hline
\end{tabular}

description would be "Fail to check patient bed area". At this stage of the analysis it is possible to make a prediction of what the consequence of that error might be. The chart would remain mislaid and because the nurse had no record of what drugs were due to be taken or when, drug doses would be missed. Thus, in the fourth column under the heading “consequences” a description of the potential consequence of the activity is entered. The fifth column of the table (recovery) indicates whether or not the error can 
be recovered. It may be that by completing further task steps, the nurse will be able to go back and correct the original error or omission. If this is the case that task step at which the original error may be recovered is entered in column 5 under the heading "Recovery". If it is not possible to recover the error then the "Recovery " column is left blank.

The probability of the error occurring and its criticality are denoted in the table by $\mathrm{P}$ and $\mathrm{C}$ respectively. The probability of an error is categorised as low (hardly ever occurs), medium (has occurred once or twice) or high (occurs frequently). Criticality (C) is usually all or none and it must be acknowledged that many drug administration errors are potentially critical. However the extent to which many administration errors cause a fatality or serious injury is highly variable and is dependent on numerous factors such as the drug's potency and therapeutic range, the age of the patient (elderly or paediatric patients are more likely to be adversely affected); the condition of the patient, serum levels, the point in the administration chain the error occurred, the nurse's knowledge of drugs or the nurse's experience. For the purposes of this assessment criticality will be modified to reflect three levels of severity: low (L), medium (M) and high (H). The levels of severity correspond to the following descriptions

L Level 0: No medication error

Level 1: Error: occurred, no harm to the patient

M Level 2: Error: increased need for monitoring, no change in vital sings

Level 3: Error: increased: monitoring, transient change in vital signs, no harm to patient

H Level 4: Error: increased monitoring, change in vital signs - treatment needed, change in length of stay or effect on an investigational drug protocol 
Level 5: Error: increased monitoring and treatment, change in patient Morbidity

Level 7: Death

(Modified from Demer and Moore, 1988)

The incidences indicated as low in criticality are likely to be recoverable by an alternative course of action. For example, being unable to find the keys to the drug trolley (2.3.1) could be overcome by ordering further supplies from the pharmacy. But this course of action may not prevent doses being missed. Putting a bottle back in the wrong place in a medicine store may cause the next person to select the wrong bottle from the shelf. A misplaced bottle might contribute to an error but in this case reading the label offers the opportunity of recovery. A more serious potential error would be to put the medicine bottle in an inappropriate place such as the IV fluids store or in the store for diagnostic tests. Such an action would cause the medication to become mislaid and would again lead to drug doses being missed.

In this generalised version of the model it is difficult to quantify criticality. Dean and Barber (1999) have devised a more accurate method for scoring drug administration errors based on specific cases drawn from literature and scored by a cross section of health professionals. The cases used refer to errors with specific drugs. As this model makes no reference to specific drugs and takes no account of patient conditions it cannot make accurate estimates of criticality.

\section{Remedial design strategies}

The final column shows the measures that could be taken to reduce errors. These are mainly in the form of design of products and technological systems. It is important to 
note that in order to be effectively implemented any design solution needs to regulated by appropriate management and organisational controls.

\section{Technology}

Radio frequency tagging was suggested as a solution to the problem of missing medication administration records. It is also a useful system for the location of equipment. Medication errors often stem from mistakes during prescribing because of slips in attention or because prescribers do not apply the relevant rules (Dean and et al, 2002). Prescription errors can be reduced by entering orders for medication into a computer system thereby solving the problems related to prescribing (bad handwriting, non-standard abbreviations, incomplete drug orders) and in particular errors related to compatibility of combined drugs or patient allergies. This solution also resolves the problem of missing charts since the patient's drug information is held in a data bank. Integrating computerised medication order entry with bar coding scanners also solves problems arising from reading the wrong drug name.

\section{Labelling}

Medical personnel often complain about the similarity of drug labels which makes it difficult to discriminate among products. Pharmacy products dispensed for named patients have a small white label printed with the patient's name, drug name, administration instructions and any warnings in a small black or grey font. The result is that very often the bottles and labels all look similar. Adding colourful or eye-catching features to the packaging or labelling would increase the nurse's capability to distinguish products. Increasing the conspicuity of product labels in this way should help to improve search strategies. For instance, highlighting salient information such as drug name and dose would enable these features to be readily picked out. This strategy 
could also be extended to storage areas so that one class of drugs is not mistaken for another. Using colour labels to distinguish categories of drugs is a common practice in anaesthesia.

\section{Equipment}

Selecting the wrong needle for a syringe assembly or the wrong tube for an IV set might be due to a genuine lack of knowledge or because of the large availability of items from which to select. The NPSA has identified the latter problem in the case of infusion devices. A pilot study revealed an average of 1,065 devices at the hospitals and an average of 31 differing types with 65\% of these unused most of the time. (NPSA, 2004). Assessing usage and limiting the variety of equipment reduces the number of errors caused by selection are made and is a good way to force individuals to make the right choice. This could be achieved by purchasing from a small number of suppliers, maintaining the same suppliers and procuring a small range of equipment in order to achieve some standardisation in available devices. This would help agency nurses and those who work on several units who are often exposed to a confusing array of different devices. Writing formal protocols for setting up and checking IV systems would be useful in addition to attaching simplified instructions to the device itself which could be seen when programming.

\section{Discussion}

The main purpose for carrying out this analysis was to demonstrate the types of error that occur during drug administration and where in the process these errors occur. The analysis predicts what steps can be taken to achieve resolution and highlights those aspects of the drug administration process where design solutions would have the greatest impact. 
Many of the tasks presented in the HTA could be subdivided into further levels of component tasks and operations thus revealing a highly detailed description of the drug administration task. The error mode taxonomy prompts the analyst to consider potentially unforeseen errors and the error reduction strategies are readily identified. The strength of the SHERPA technique is that it can be used to analyse tasks or processes at many different levels. The technique could be adapted to different ward settings and could also be applied to a range of different health care procedures. For instance, the method could be used to analyse equipment usage in order to highlight training requirements or to re-draft protocols as part of the quality review process.

One disadvantage of using SHERPA as an error prediction tool is that a task analysis has to be drawn up before error predictions can be made. In order to gain a full description of every step of the drug administration task, several long HTAs would be required. Thus to obtain a high level of detail could be time-consuming, particularly in cases where no formal protocols exist. Another weakness of SHERPA is that actions considered highly unlikely are excluded from the analysis. An example of such exclusion occurs at step 3.3.2 of the HTA ("hang an infusion bag”) as part of setting up an IV infusion. Failing to hang an IV bag would be considered by most nurses as an impossible error to make. But if the situation arose where there was a shortage of infusion stands or the infusion bag had been removed from the stand whilst the patient was being moved, then this situation would not be so improbable. However, failing to hang the IV bag due to a shortage of stands would constitute a systems error and thus this error mode should be included in the SHERPA analysis. 
Kirwan (1992) compared SHERPA with 5 other human error identification techniques on the criteria of comprehensiveness, accuracy, consistency, theoretical validity, usefulness, resource usage and auditability/acceptability to see if the incidents predicted by the human error identification techniques matched those that had actually occurred. SHERPA performed well against the other techniques and achieved a high ranking by the analysts. Stanton and Stevenage (1998) demonstrated that participants using SHERPA correctly predicted more errors and missed fewer errors than those using heuristics. However they also wrongly predicted more errors. When in a second study participants applied the SHERPA method to the task on three separate occasions there was little change in the numbers of hits and misses. The frequency of false alarms fell over time producing an apparent increase in the number of correct rejections.

The task analysis could be extended to take account of the activities occurring during the administration of controlled drugs, injections that have to be reconstituted or those whose dose has to be calculated according to the patient's weight, of medications with particular emphasis on those that need to be reconstituted or those that need to be administered according to the patient's weight. The overall technique could be used to compare different systems of administering medication. Validation of the errors predicted in the model need to be compared with data gathered by observation of 'realworld' drug administration. This simplified model excludes the condition of the patient and the routine checks (blood pressure, temperature, kidney function) that are carried out on a regular basis. The model would benefit from inclusion of patient states, as these have direct bearing on how nurses respond.

Medication administration errors occur due to a number of varying and often interacting factors that may originate from organisational practices, the working environment or 
personal and professional practices. There is an underlying assumption that the SHERPA taxonomy is able to capture the full range of error producing activity whereas this is not the case. Communication with patients and their relatives, colleagues and various departments all impinge on the process of drug administration. These factors cannot be analysed effectively using the taxonomy and would require other techniques.

Successful drug administration is dependent on an effective patient information or patient monitoring system. Some drugs have a limited therapeutic range and various indicators (such as blood pressure, heart rate, anticoagulant levels) have to be checked before they can be administered in the appropriate quantities. Medical staff also need to know which drug is needed and when. This cannot be accurately ascertained in the absence of the medication chart.

Solutions based on computerised technologies must be introduced only after carrying out a full and detailed analysis of all existing systems. This is because of high cost of implementation, changes in working patterns and compatibility with other hospital departments. Computer technology has been demonstrated to reduce medication errors however care should be taken to ensure that its use does not create latent errors elsewhere in the system.

This paper illustrates how a human factors techniques can be applied to the process of administering drugs to hospitalised patients as a means of preventing error or reducing the effects of error. Schnieder (2002) advocates the benefits of applying human factors to the issue of medication errors and makes the point that healthcare providers need to be more aware of the limits of human performance and that system changes need to be made to accommodate these limits. Whilst this paper considers the actions of an 
individual nurse in the process of administering drugs, the authors recognise that, as noted above, that drug administration errors are rarely entirely due to the actions of a single individual. There is still an expectation of perfection among medical professionals at a time when risk is an accepted part of daily life in other fields of activity. This is due to training and a number of other factors such as the culture of blame and the punitive measures that still exists in the NHS. The blame culture is slowly changing largely due to several patient safety initiatives set up by the Department of Health and through the activities of the National Patient Safety Agency.

\section{References}

Anderson, D.J., Webster, C.S., 2001. A systems approach to the reduction of medication error on the hospital ward. Journal of Advanced Nursing, 35: (1), 34-41.

The Audit Commission, 2001. A Spoonful of Sugar, London.

Bates, DW, Cullen, D., Laird, N., Petersen, L.A., Small, S.D., Servi, D., et al 1995. Incidence of adverse drug events and potential adverse drug events: implications for prevention. Journal of the American Medical Association; 274, 29-34.

Bates DW, Teich J. Lee Seger, D,, Kuperman, G.J,, Boyle, D. et al, 1999. The importance of computerized physician order entry on medication error prevention. Journal of the American Medical Informatics Association, 6, 313-321.

Bates, D.W., 2000. Using information technology to reduce rates of medication errors in hospitals. British Medical Journal, 320, 788-791.

Brennan, T. A., Leape, L.L, Lair, N.M., Herbert, L., Localio, A.R. et al. 1991. Incidence of adverse events and negligence in hospitalised patients. New England Journal of Medicine, 324, 370-376.

Cohen, M. R., Senders, J., Davis, N.M., 1994. Failure mode and effects analysis: A model approach to avoiding dangerous medication errors and accidents. Hospital pharmacist, 29, 319-328.

Croteau, R.J., Schyve, P.M., 2000. Proactively Error-Proofing Health Care Processes. In Spath P (ed) Error reduction in Health care: A systems approach to improving patient Safety. Jossey-Bass, San Fransisco.

Dean, N., Barber, et al. 2000. Prescribing errors in hospital inpatients: why do they occur? The Pharmaceutical Journal 265, 7114 **. 
Dean, B.S. and Barber, N.D. (1999), A validated, reliable method of scoring the severity of medication errors. American Journal of Health System Pharmacy, 56, 57-61.

Dean, B., Schachter, M., et al 2002. Causes of prescribing errors in hopsital inpatients: a prospective study. The Lancet, 359, 1373- 137.

Department of Health, "An organisation with a memory: report of an expert group on learning from adverse events In the NHS. London: DoH, 2000.

Ferner, R. E., 1995. Misleading drug packaging. British Medical Journal (Clinical Research Ed.) 311, 7003, 514-514.

Fiesta, J., 1998. Failure to communicate. Nurs.Manage. 29, (2), 22-25.

Hospital Health Network. 2001. Medication safety issue brief. Using automation to reduce errors. Part 2. Feb; 75(2):33-4.

Kirwan B., (1994). A Guide to Practical Human Reliability Assessment. London: Taylor and Francis.

Kirwan, B., Validatin of human reliability assessment techniques: part 1- Validation issues. Safety Science, vol 27, 25-41f

Kohn, L.T., Corrigan, J.M., Donaldson, M.S. (Eds.), 1999. To err is human:Building a Safer Health System. Washington, DC: National Academy Press.

Leape. LL, Bates, D.W., Cullen, D.J., Cooper, J., Demonaco, H.J., Gallivan T, Hallisey R, Ives J, Laird, N, Laffel, G., Nameskal, R, Petersen, L.A, Porter, K., Servi D, Shea, B.F., Small, S.D., Sweitzer, B.J., Thompson, B.T., Vliet, M.V., 1995, Systems analysis of adverse drug events. Journal of the American Medical Association, 274: (1), 35-43.

Leape, L.L., Brennan, T.A., Laird, N.M., Lawthers, A.G., Localio AR, Barnes BA, et al. 1991. The nature of adverse events in hospitalized patients: results from the Harvard Medical Practice Study. II. N Engl J Med; 324: 377-384.

Leape, L.L., Berwick, D., 2000. Safe Healthcare: are we up to it? British Medical Journal, 320: pp 725-726.

McNutt, R.A, Abrams, R., Aron, D.C., 2002. Patient Safety efforts should focus on medical errors. Journal of the American Medical Association., 287: (15), 199.

NPSA

Phillips J., Beam, S., Brinker, A., Holquist, C., Honig, P., Lee „L.Y. Pamer, C., 2001. Retrospective analysis of mortalities associated with medication error. American Journal of Health System Pharmacy, 58: 1835-1841.

Taxis, K., Dean, B.A., .Barber, N.D., 1999. Hospital drug distributions system in the UK and Germany - a study of medication errors. Pharmacy World Science, 21: 25-31. 
Thornton, P.D., Simon, S., Matthew, T.H.,1999. Towards safer drug prescribing, dispensing and administration in hospitals. Journal of Quality in Clinical Practice, 19: 41-45.

Schneider, P.J., 2002. Applying human factors in improving medication-use safety. American Journal of Health Systems Pharmacy 59 (12), 1155 - 1159.

Stanton, N. and Stevenage, S. Applied Ergonomics 


\section{Drug Administration Hierarchical Task Analysis}

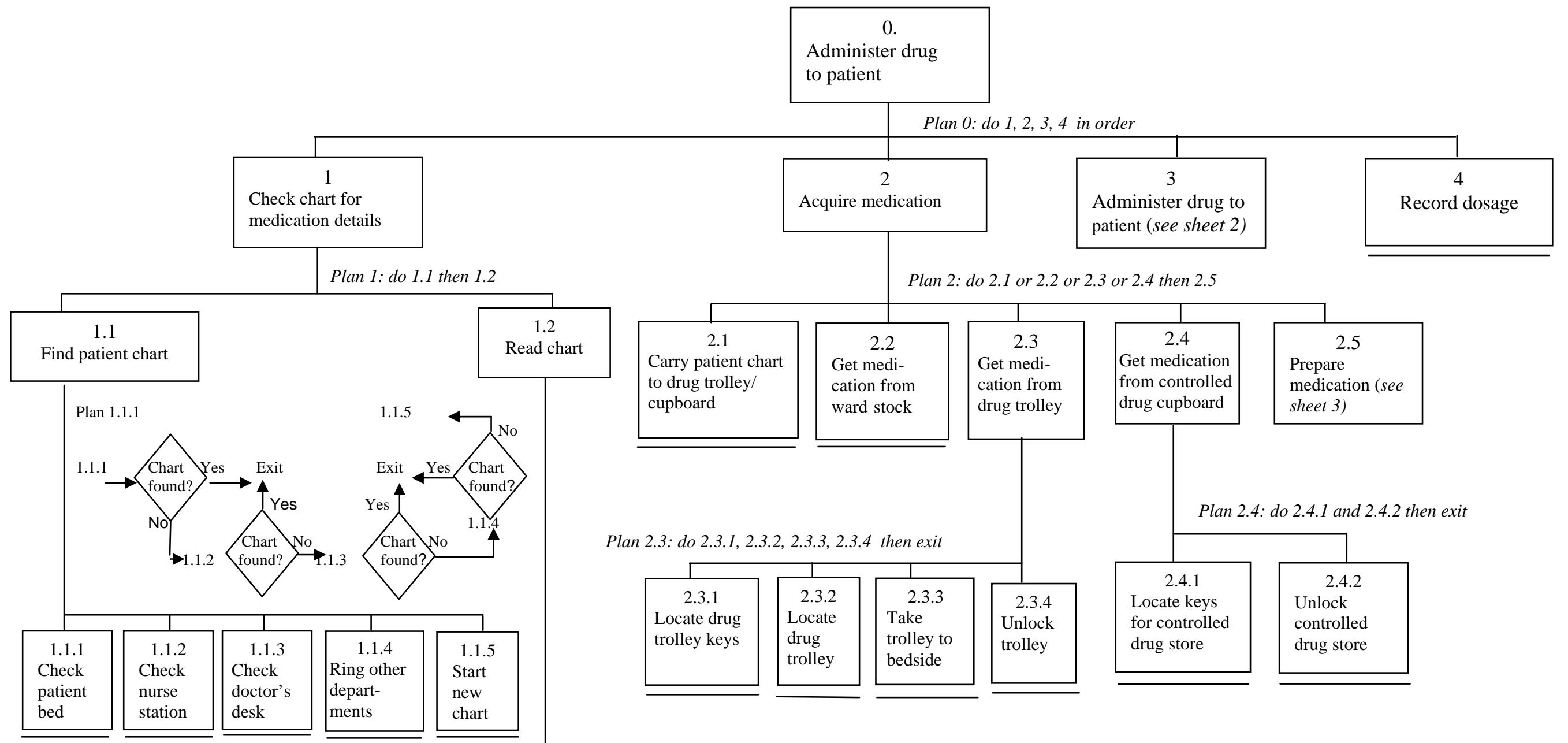

Plan 1.2: do 1.2.1, 1.2.2, 1.2.3, 1.2.4, 1.2.5, 1.2 .6 exit

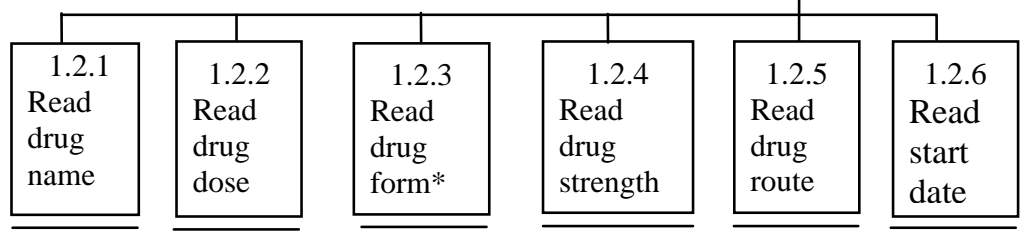

* Pharmacuetical form eg plain, soluble or slow release tablets 


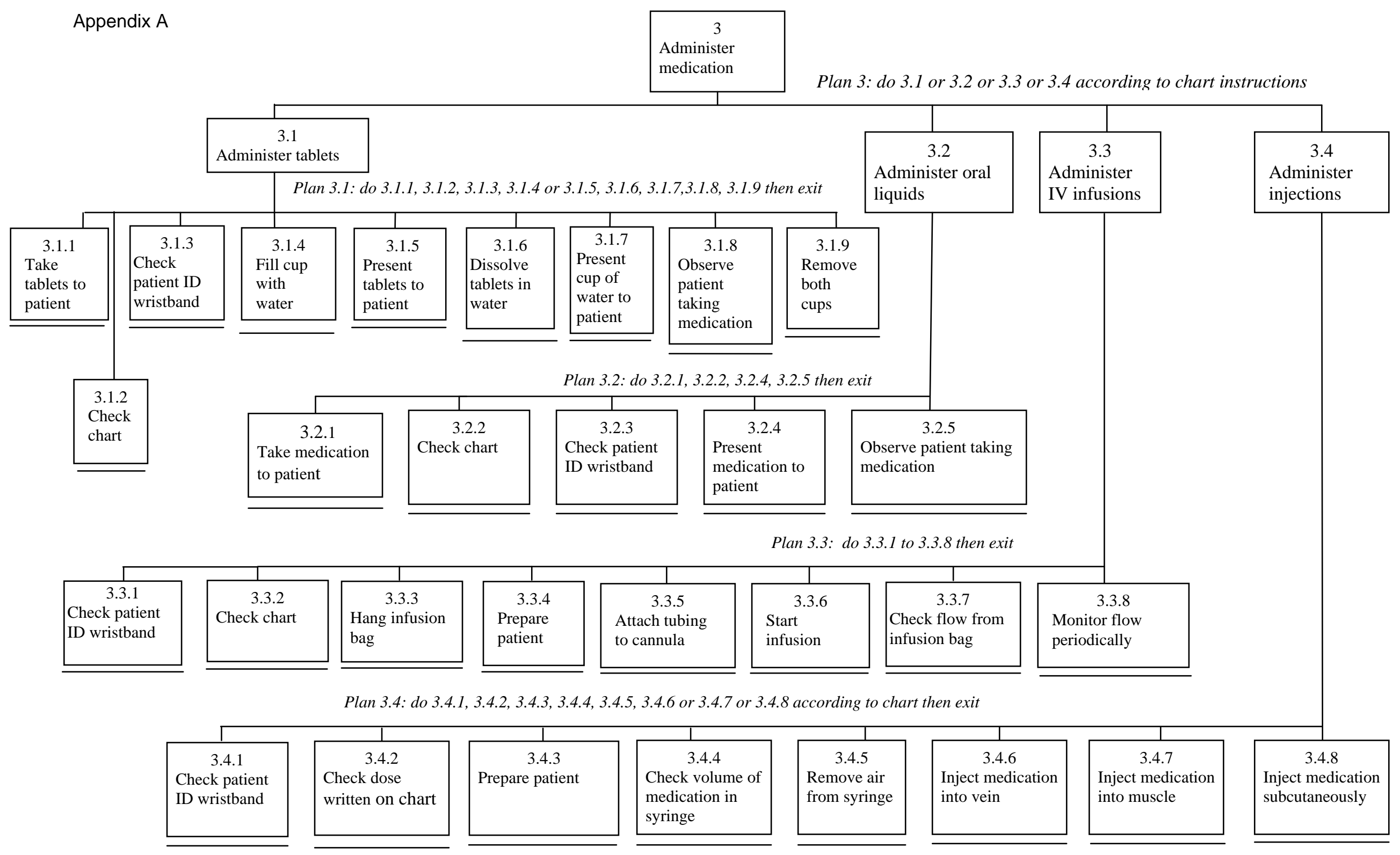




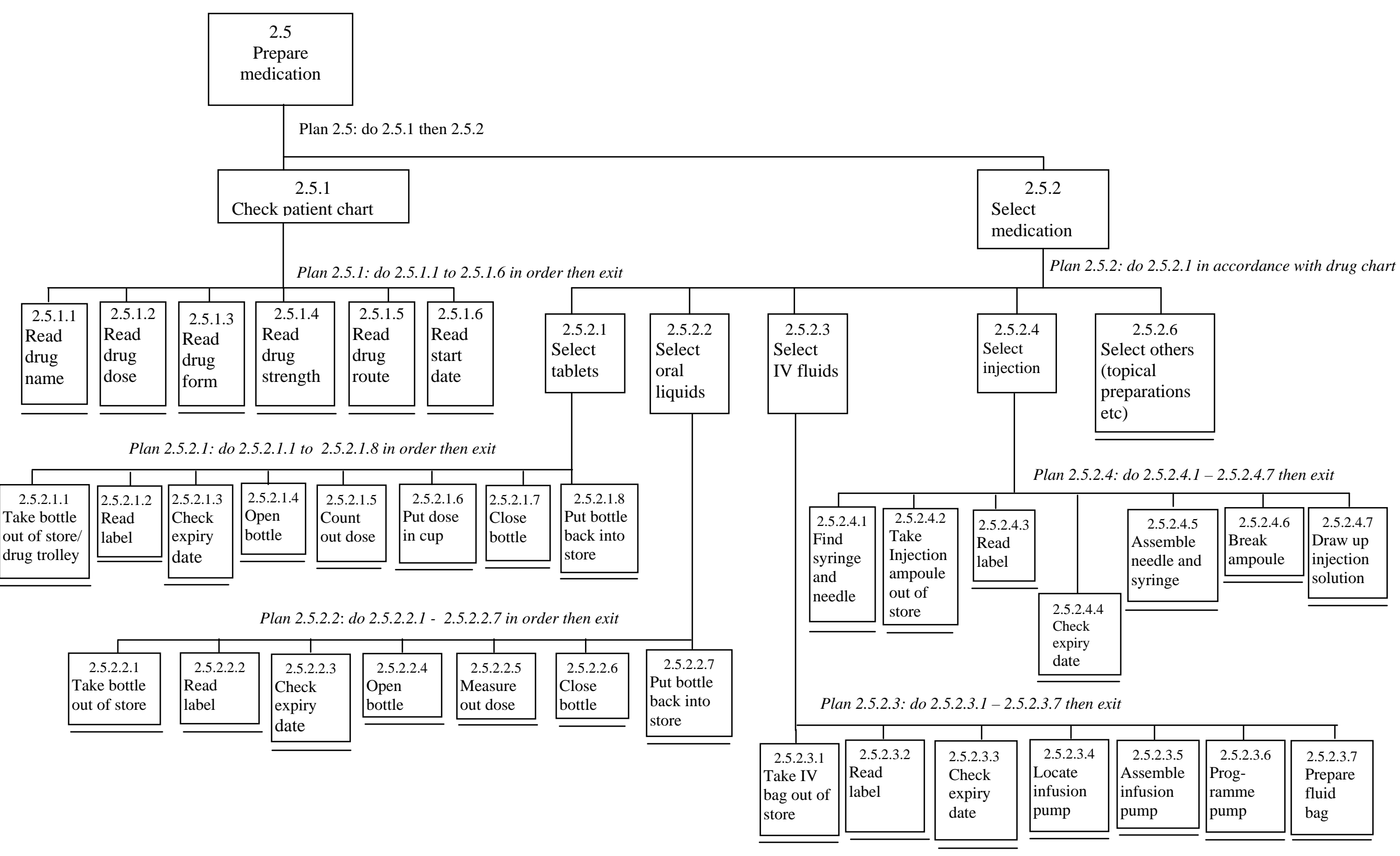


SHERPA Output - Human Error Analysis Table

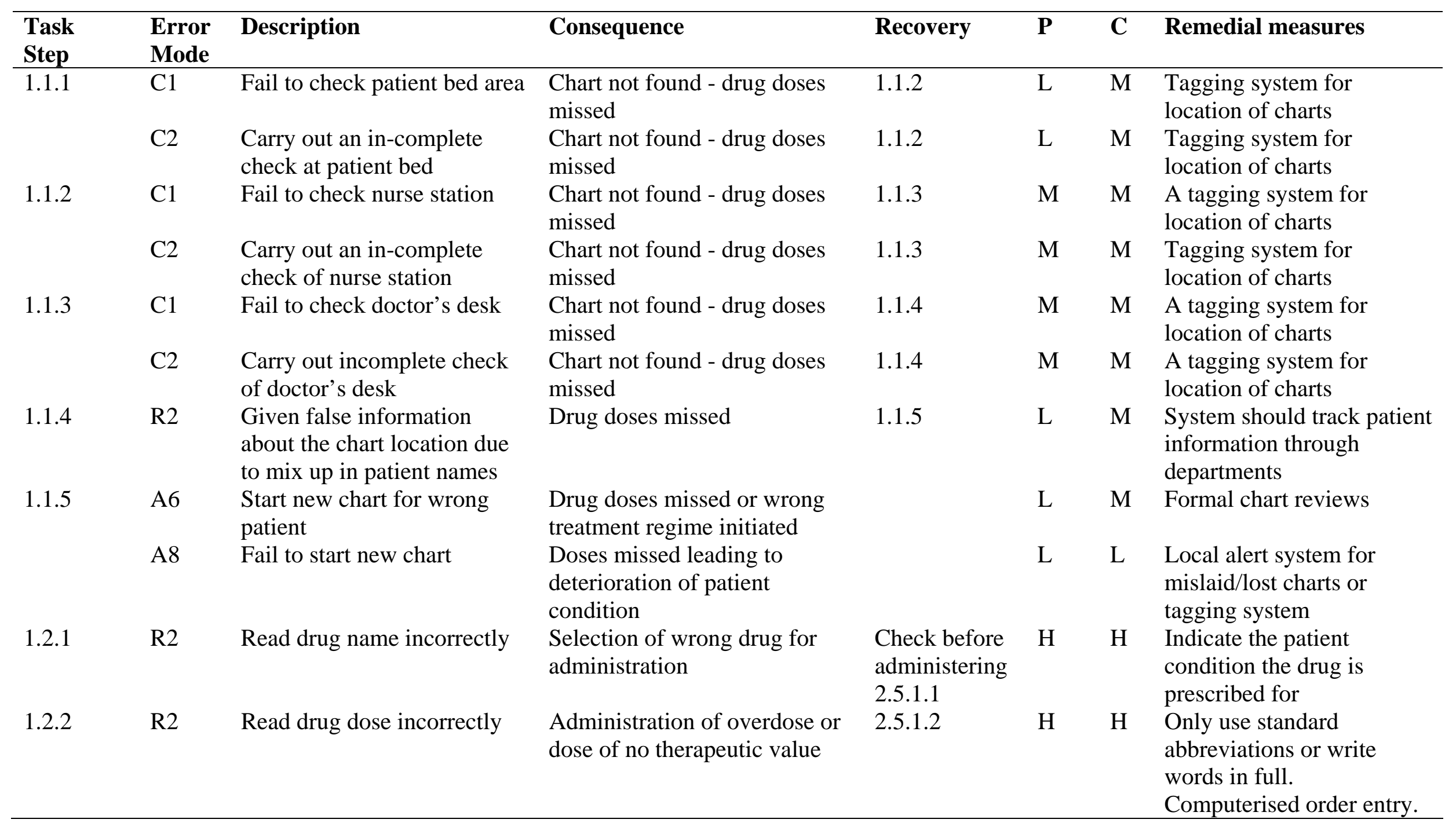




\begin{tabular}{|c|c|c|c|c|c|c|c|}
\hline $\begin{array}{l}\text { Task } \\
\text { Step }\end{array}$ & $\begin{array}{l}\text { Error } \\
\text { Mode }\end{array}$ & Description & Consequence & Recovery & $\mathbf{P}$ & $\mathbf{C}$ & Remedial measures \\
\hline 1.2 .4 & $\mathrm{R} 1$ & Fail to read drug strength & $\begin{array}{l}\text { Administer an overdose or } \\
\text { dose of no therapeutic value }\end{array}$ & 2.5.1.4 & M & $\mathrm{H}$ & $\begin{array}{l}\text { Computerised order entry } \\
\text { coupled with barcodes and } \\
\text { scanning }\end{array}$ \\
\hline 1.2.6 & $\mathrm{R} 1$ & Fail to read start date & $\begin{array}{l}\text { Give drug too early/late in } \\
\text { treatment }\end{array}$ & 2.5.1.6 & $\mathrm{L}$ & $\mathrm{L}$ & $\begin{array}{l}\text { Highlight or write } \\
\text { conspicuously }\end{array}$ \\
\hline 2.1 & A8 & $\begin{array}{l}\text { Fail to carry chart to drug } \\
\text { trolley or drug storage area }\end{array}$ & $\begin{array}{l}\text { May forget drug name, dose, } \\
\text { form, route }\end{array}$ & None & M & $\mathrm{L}$ & $\begin{array}{l}\text { Take chart to drug } \\
\text { store/trolley }\end{array}$ \\
\hline 2.2 & A6 & $\begin{array}{l}\text { Get wrong medication from } \\
\text { ward stock }\end{array}$ & Give wrong drug & 2.5.1.1 & $\mathrm{H}$ & $\mathrm{H}$ & $\begin{array}{l}\text { Better labelling in storage } \\
\text { area. Greater conspicuity } \\
\text { of labels }\end{array}$ \\
\hline 2.3 .2 & A9 & Fail to locate drug trolley & Doses omitted & & $\mathrm{L}$ & $\mathrm{H}$ & $\begin{array}{l}\text { Keep trolley in designated } \\
\text { place. Tagging }\end{array}$ \\
\hline 2.4 .1 & A9 & $\begin{array}{l}\text { Unable to find controlled } \\
\text { drug cupboard keys }\end{array}$ & Doses omitted & & $\mathrm{L}$ & $\mathrm{H}$ & $\begin{array}{l}\text { Keep trolley in designated } \\
\text { place. Tagging }\end{array}$ \\
\hline 2.5.1.1 & $\mathrm{R} 2$ & Read drug name incorrectly & $\begin{array}{l}\text { Selection of wrong drug for } \\
\text { administration }\end{array}$ & 2.5.2.1.2 & $\mathrm{H}$ & $\mathrm{H}$ & $\begin{array}{l}\text { Indicate the patient } \\
\text { condition the drug is } \\
\text { prescribed for }\end{array}$ \\
\hline
\end{tabular}




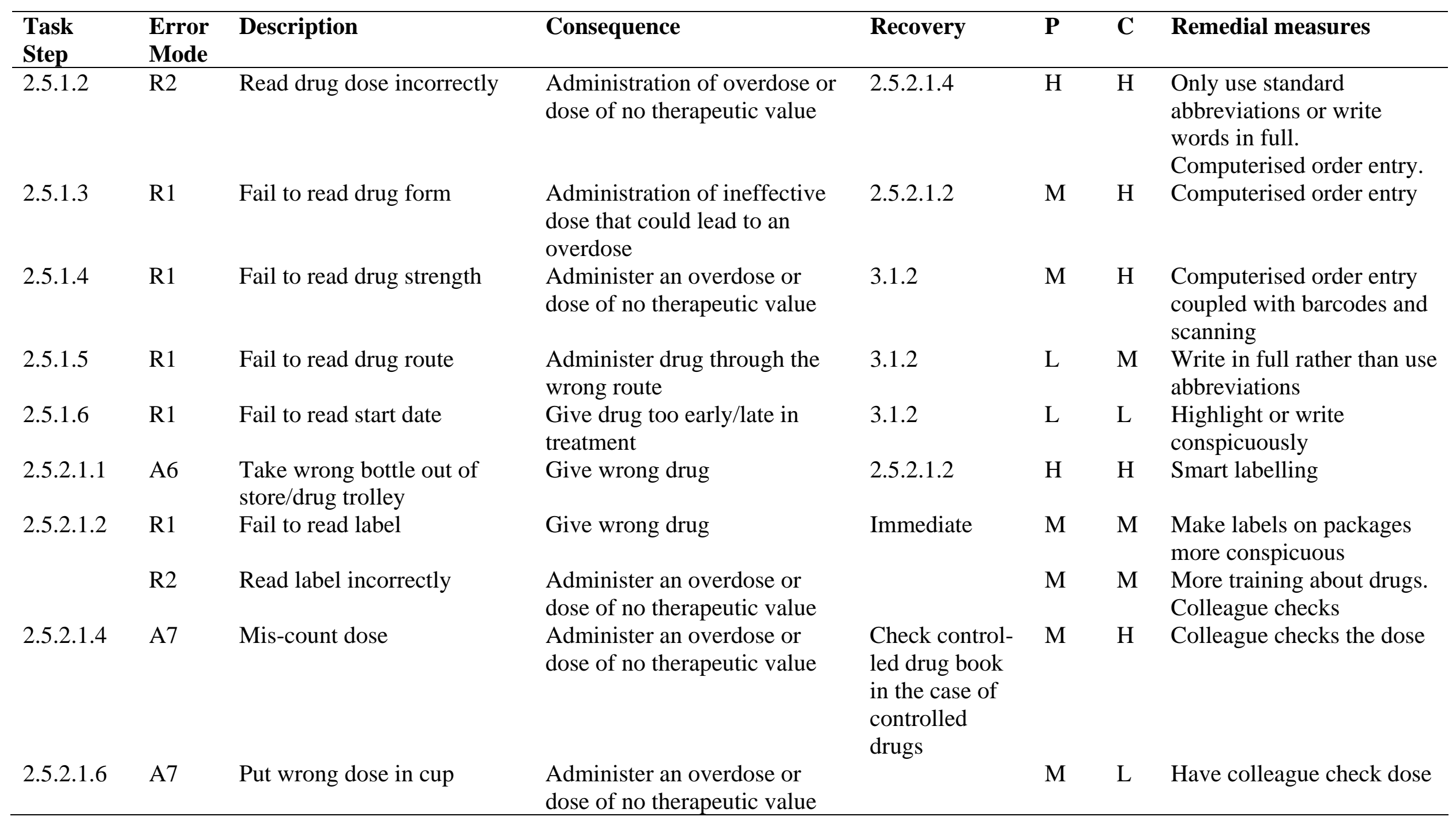




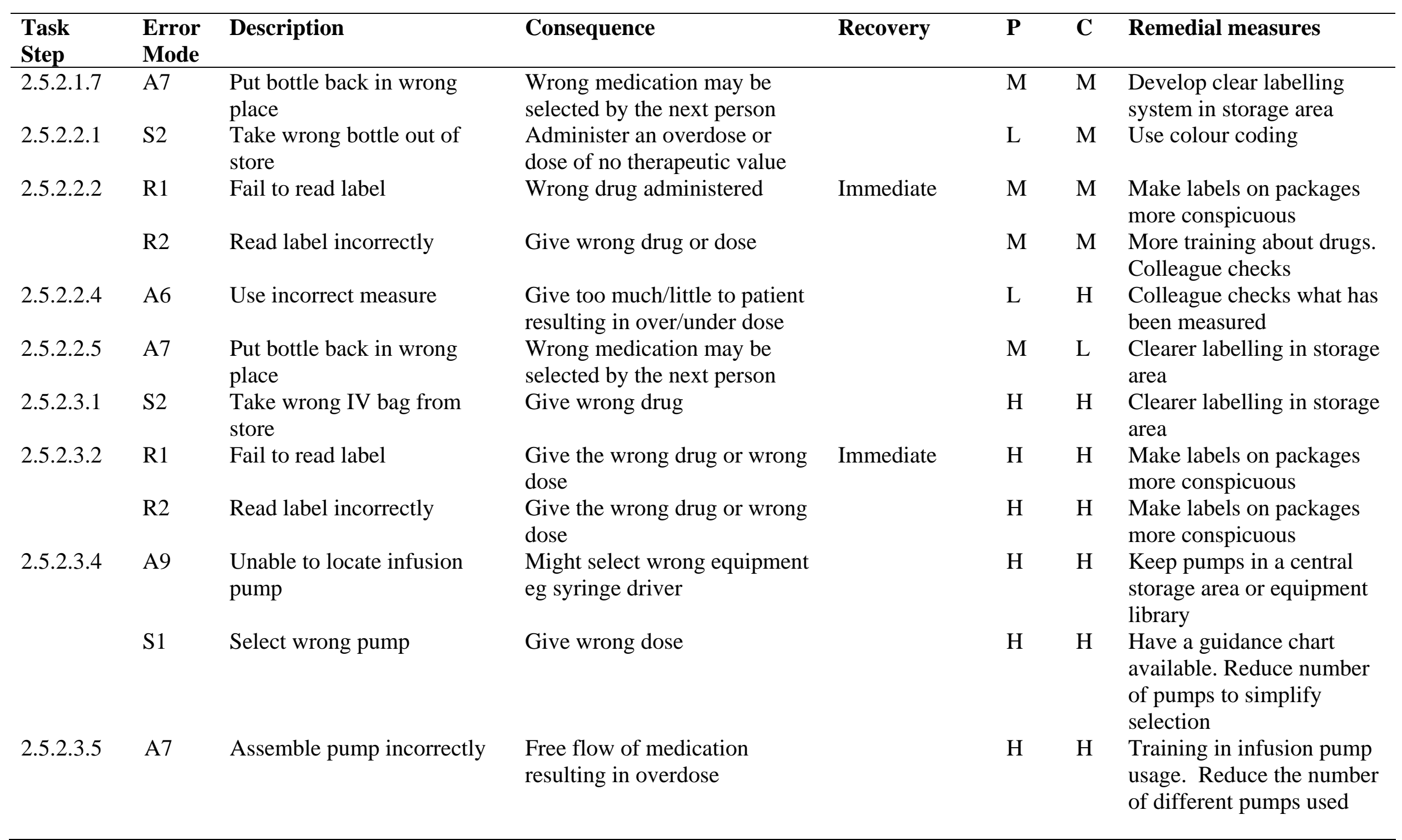




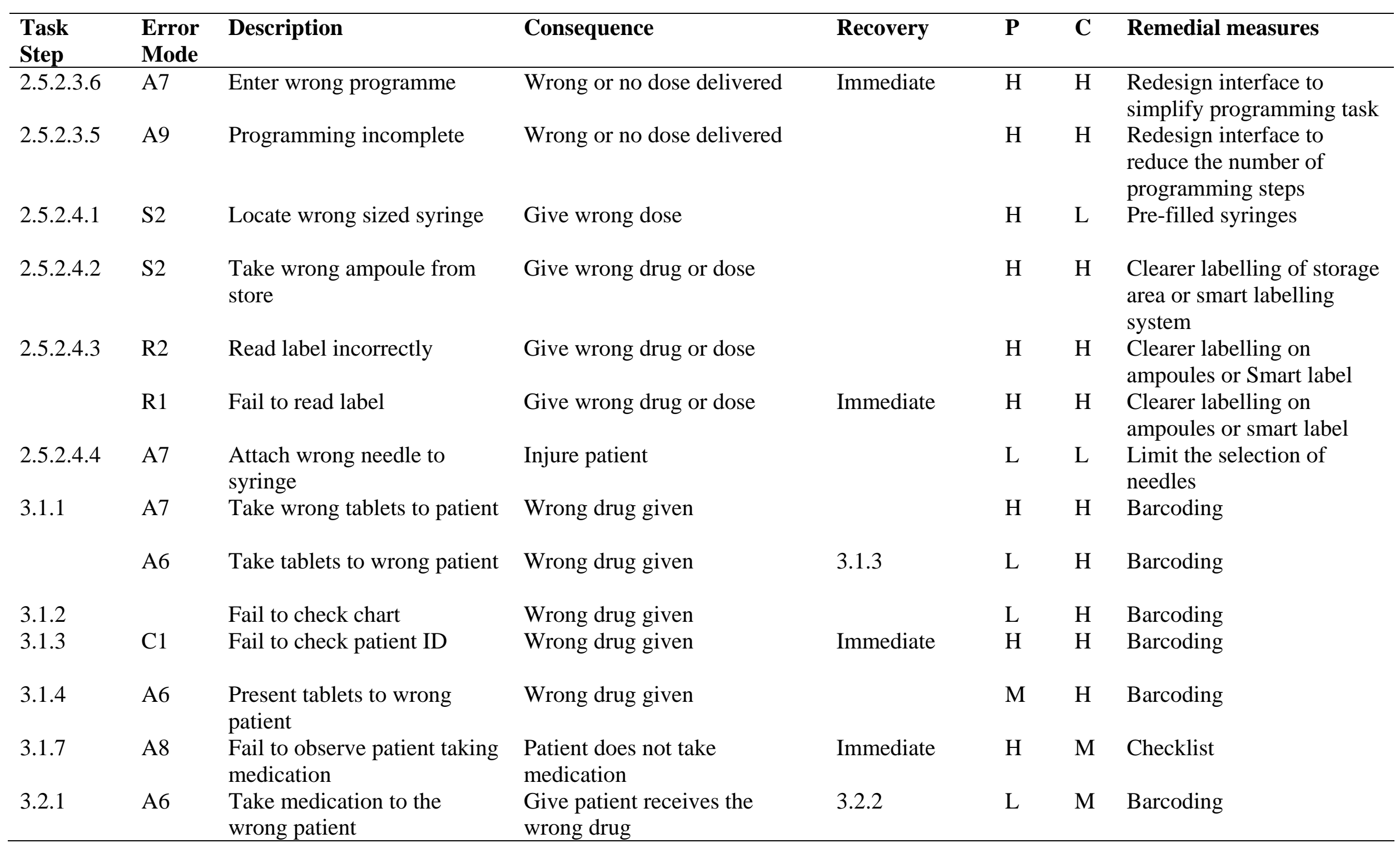




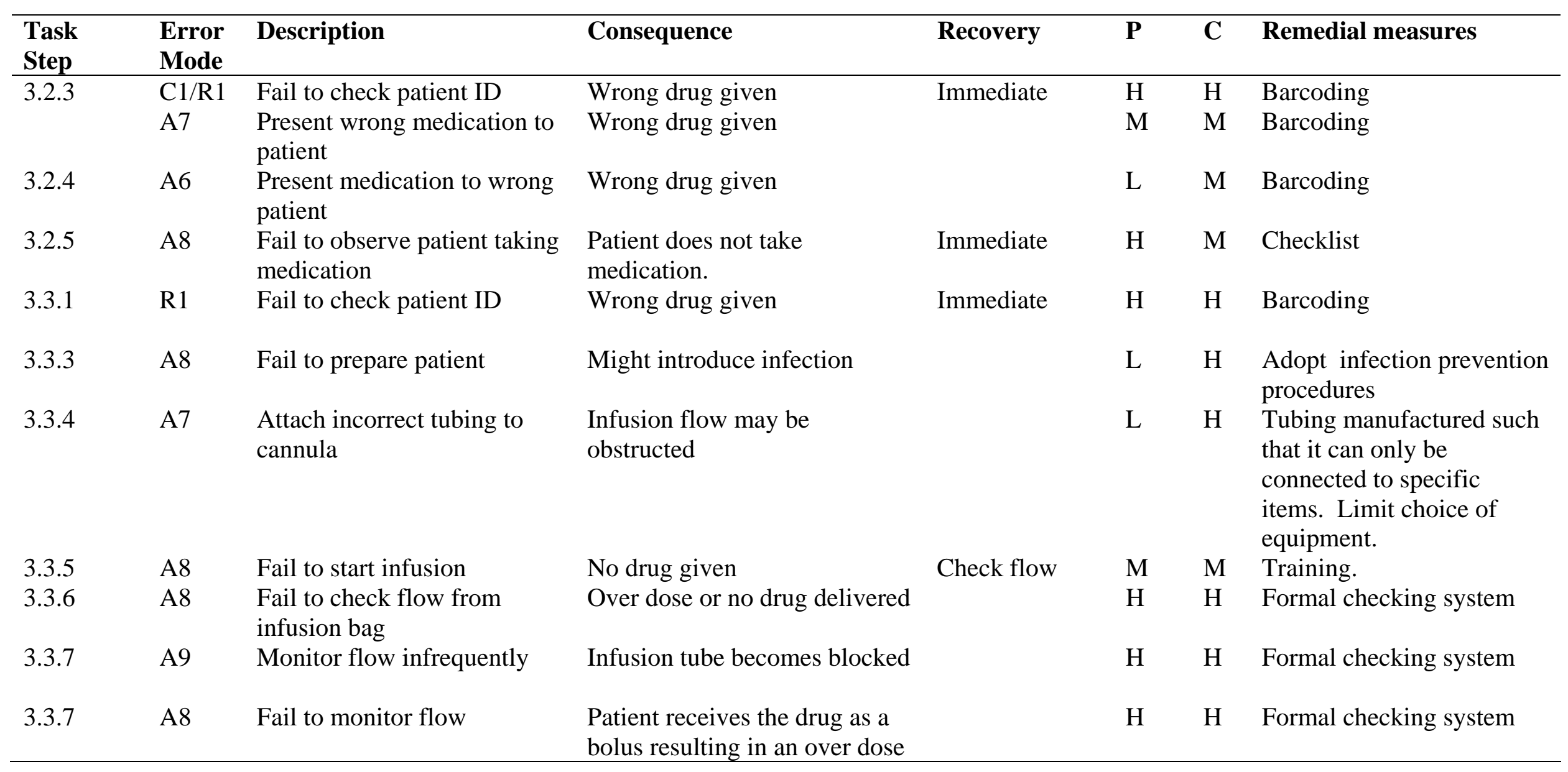




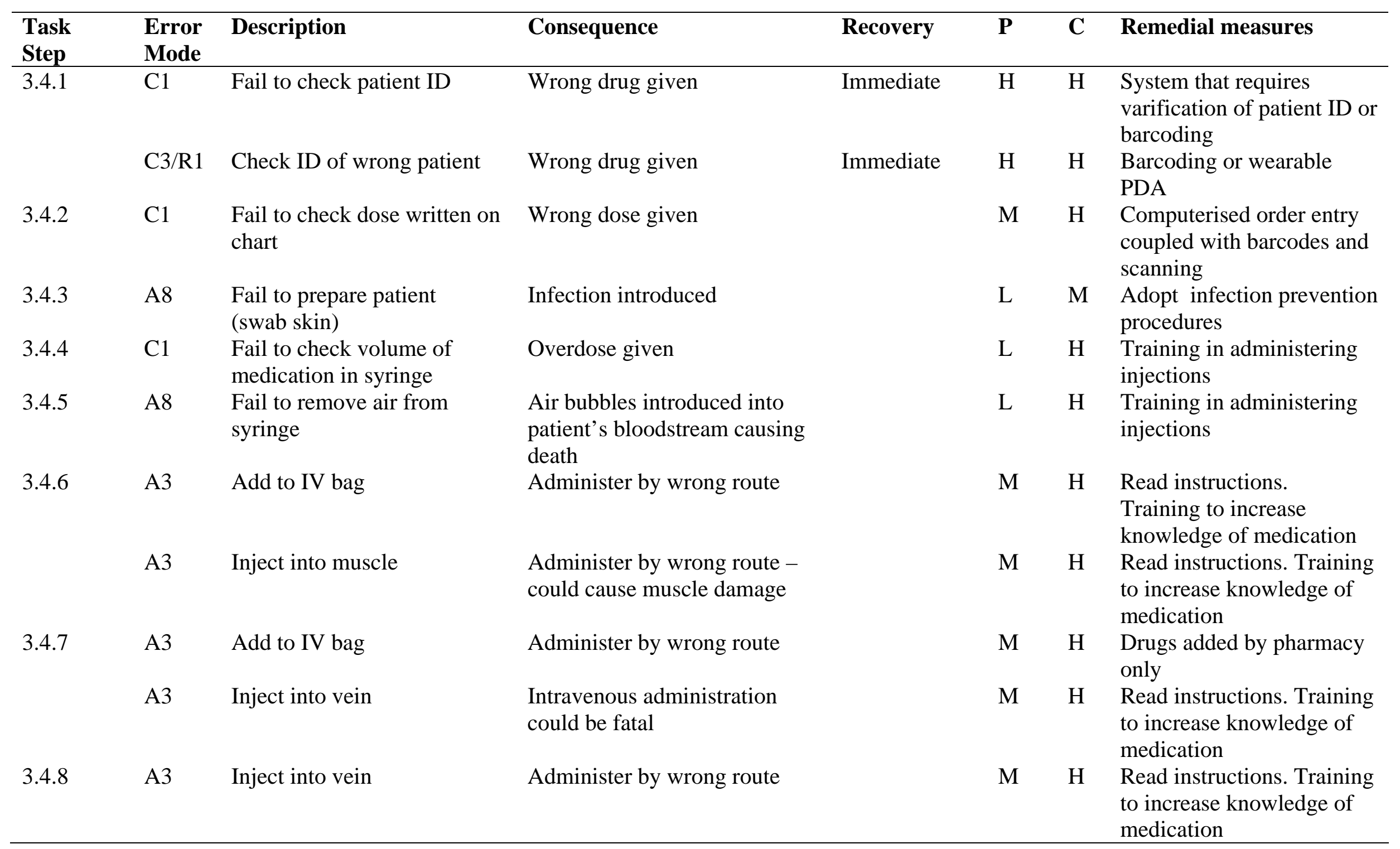


